\title{
CERTAIN FUNCTIONAL RELATIONS FOR THE DOUBLE HARMONIC SERIES RELATED TO THE DOUBLE EULER NUMBERS
}

\author{
HIROFUMI TSUMURA
}

(Received 6 February 2004; revised 2 December 2004)

Communicated by W. W. L. Chen

\begin{abstract}
In this paper, we give certain analytic functional relations for the double harmonic series related to the double Euler numbers. These can be regarded as continuous generalizations of the known discrete relations obtained by the author recently.

2000 Mathematics subject classification: primary 40B05; secondary $11 \mathrm{M} 41$.

Keywords and phrases: double Euler numbers, double harmonic series, Mordell-Tornheim double zeta functions, Riemann zeta function, uniformly convergent series.
\end{abstract}

\section{Introduction}

Let $\mathbb{N}$ be the set of natural numbers, $\mathbb{N}_{0}=\mathbb{N} \cup\{0\}, \mathbb{Z}$ the ring of rational integers, $\mathbb{Q}$ the field of rational numbers, $\mathbb{R}$ the field of real numbers, and $\mathbb{C}$ the field of complex numbers.

As multiple analogues of the Tornheim double series, Matsumoto defined the Mordell-Tornheim zeta function

$$
\zeta_{M T, r}\left(s_{1}, s_{2}, \ldots, s_{r} ; s\right)=\sum_{m_{1}, m_{2} \ldots \ldots m_{r}=1}^{\infty} \frac{1}{m_{1}^{s_{1}} m_{2}^{s_{2}} \cdots m_{r}^{s_{r}}\left(m_{1}+\cdots+m_{r}\right)^{s}}
$$

for complex variables $s_{1}, s_{2}, \ldots, s_{r}, s$, where the sum is over $r$-tuples of positive integers (see [3]). He showed that this function can be continued meromorphically to

Partially supported by Grant-in-Aid for Science Research (No. 17540053), the Ministry of Education, Culture, Sports, Science and Technology, Japan.

(C) 2005 Australian Mathematical Society $1446-7887 / 05 \$ \mathrm{~A} 2.00+0.00$ 
$\mathbb{C}^{r+1}$. In the 1950's, the values $\zeta_{M T, 2}\left(k_{1}, k_{2} ; k\right)$ for $k_{1}, k_{2}, k \in \mathbb{N}$ were investigated by Tornheim [6] and Mordell [4]. After that, various explicit evaluation formulas for them were obtained (see, for example, $[2,5,8]$ ). Recently, as continuous generalizations of the discrete relations in [8], the author has given certain functional relations for $\zeta_{M T, 2}\left(s_{1}, s_{2} ; s\right)$, which essentially include both Tornheim's and Mordell's results (see [7]).

More recently we considered the multiple Euler numbers and the related multiple series, and obtained some relations for the values of them at positive integers (see [10]).

In the present paper, we consider

$$
\mathfrak{T}_{a, b}\left(s_{1}, s_{2} ; s\right)=\sum_{m, n=0}^{\infty} \frac{1}{(2 m+a)^{s_{1}}(2 n+b)^{s_{2}}(2 m+2 n+a+b)^{s}}
$$

for $a, b \in\{1,2\}$. Note that $\mathfrak{T}_{1.1}\left(s_{1}, s_{2} ; s\right)$ is what we considered in $[9,10]$. The aim of this paper is to give certain functional relations between these double series and the Riemann zeta function $\zeta(s)$ (see Theorem 4.7), by the same method as in [7]. For example, we have

$$
\begin{aligned}
& \mathcal{T}_{1,1}(2, s ; 3)+\mathfrak{T}_{1,2}(2,3 ; s)-\mathfrak{T}_{1,2}(s, 3 ; 2) \\
& \quad=\frac{5}{2}\left(1-2^{-s-3}\right) \zeta(s+3) \zeta(2)-4\left(1-2^{-s-5}\right) \zeta(s+5)
\end{aligned}
$$

for all $s \in \mathbb{C}$ with $\operatorname{Re}(s) \geq 0$. In particular, putting $s=2$ in (3), we have a non-trivial relation

$$
\mathcal{T}_{1,1}(2,2 ; 3)=\frac{155}{64} \zeta(5) \zeta(2)-\frac{127}{32} \zeta(7) .
$$

Indeed, this is a concrete example derived from [10, Theorem 1.1] (see also [9]). Thus we can regard our present results as analytic generalizations of our previous discrete results in $[9,10]$.

\section{Preliminaries}

We make use of the same notation as in $[7,9,10]$. For $u \in[1,1+\delta]$, we define a set of numbers $\left\{\mathcal{E}_{m}(u)\right\}$ by

$$
F(x ; u)=\frac{2 u e^{x}}{e^{2 x}+u}=\sum_{m=0}^{\infty} \varepsilon_{m}(u) \frac{x^{m}}{m !} .
$$


We denote $\mathcal{E}_{m}(1)$ by $E_{m}$ which is called the $m$ th Euler number (see [1, Chapter 1]). We see that (see $[9$, Section 2])

$$
\begin{gathered}
\mathcal{E}_{2 j+1}(1)=E_{2 j+1}=0 \quad\left(j \in \mathbb{N}_{0}\right), \\
\liminf _{m \rightarrow \infty}\left(\frac{\left|\mathcal{E}_{m}(b ; u)\right|}{m !}\right)^{-1 / m} \geq \frac{\pi}{2} \quad(u \in[1,1+\delta]) .
\end{gathered}
$$

For $s \in \mathbb{C}$ with $\operatorname{Re}(s)>0$, we define

$$
\rho(s ; u)=\sum_{m=0}^{\infty} \frac{(-u)^{-m}}{(2 m+1)^{s}} .
$$

LEMMA 2.1. For $u \in[1,1+\delta], \rho(s ; u)$ is defined and holomorphic for all $s \in \mathbb{C}$. Let $c \in \mathbb{R}$ with $0 \leq c<1$. For any $\gamma \in \mathbb{R}$ with $0<\gamma<\pi / 2$, there exists a constant $M=M(\gamma)>0$ independent of $u \in[1,1+\delta]$ such that

$$
\frac{|\rho(c-n ; u)|}{n !} \leq \frac{M(\gamma)}{\gamma^{n}} \quad\left(n \in \mathbb{N}_{0}\right)
$$

In particular,

$$
\liminf _{n \rightarrow \infty}\left(\frac{|\rho(c-n ; u)|}{n !}\right)^{-1 / n} \geq \frac{\pi}{2}
$$

PROOF. Let $\gamma \in \mathbb{R}$ with $0<\gamma<\pi / 2$, and $C_{\gamma}: z=\gamma e^{i t}$ for $0 \leq t \leq 2 \pi$, where $i=\sqrt{-1}$. We can easily check that

$$
\int_{C_{\gamma}} F(z ; u) z^{-n-1} d z=\frac{(2 \pi i) \mathcal{E}_{n}(u)}{n !} \quad\left(n \in \mathbb{N}_{0}\right) .
$$

Let $M_{1}(\gamma)=\max |F(z, u)|$ for $(z, u) \in C_{\gamma} \times[1,1+\delta]$. Then we obtain

$$
\frac{\left|\mathcal{E}_{n}(u)\right|}{n !} \leq \frac{1}{2 \pi} \int_{C_{\gamma}}|F(z ; u)||z|^{-n-1}|d z| \leq \frac{M_{1}(\gamma)}{\gamma^{n}} \quad\left(n \in \mathbb{N}_{0}\right) .
$$

Now we use the method of contour integrals (see, for example, [11, Proof of Theorem 4.2]). We consider the path $\Omega$ which consists of the positive real axis $[\gamma, \infty]$ (top side), a circle $C_{\gamma}$ around 0 of radius $\gamma$, and the positive real axis $[\gamma, \infty]$ (bottom side), where $0<\gamma<\pi / 2$. Note that we interpret $t^{s}$ to mean $\exp (s \log t)$, where the imaginary part of $\log t$ varies from 0 (on the top side of the real axis) to $2 \pi$ (on the bottom side). Let

$$
\begin{aligned}
H(s ; u) & =\int_{\Omega} F(-t ; u) t^{s-1} d t \\
& =\left(e^{2 \pi i s}-1\right) \int_{\gamma}^{\infty} F(-t ; u) t^{s-1} d t+\int_{C_{\gamma}} F(-t ; u) t^{s-1} d t .
\end{aligned}
$$


Note that $H(s ; u)$ is defined and holomorphic for all $s \in \mathbb{C}$. For $s \in \mathbb{C}$ with $\operatorname{Re}(s)>1$, letting $\gamma \rightarrow 0$, we have

$$
\begin{aligned}
H(s ; u) & =\left(e^{2 \pi i s}-1\right) \int_{0}^{\infty} F(-t ; u) t^{s-1} d t \\
& =2\left(e^{2 \pi i s}-1\right) \Gamma(s) \rho(s ; u) .
\end{aligned}
$$

Hence

$$
\rho(s ; u)=\frac{1}{2\left(e^{2 \pi i s}-1\right) \Gamma(s)} H(s ; u)=\frac{\Gamma(1-s)}{4 \pi i e^{\pi i s}} H(s ; u) .
$$

Combining (8) and (12), we see that $\rho(s ; u)$ is defined and holomorphic for all $s \in \mathbb{C}$. For $N \in \mathbb{N}_{0}$, we have

$$
H(c-N ; u)=\left(e^{2 \pi i c}-1\right) \int_{\gamma}^{\infty} F(-t ; u) t^{c-N-1} d t+\int_{C_{\gamma}} F(-t ; u) t^{c-N-1} d t .
$$

Assume $N>1$. For simplicity, we denote by $I_{1}$ and $I_{2}$ the first and second terms on the right-hand side, respectively. Note that

Hence we have

$$
\left|\int_{\gamma}^{\infty} e^{-(2 n+1) t} t^{c-N-1} d t\right| \leq \frac{e^{-(2 n+1) \gamma} \gamma^{c-N-1}}{2 n+1}
$$

$$
\left|I_{1}\right| \leq \gamma^{c-N-1}\left|e^{2 \pi i c}-1\right| \sum_{n=0}^{\infty} \frac{e^{-(2 n+1) \gamma}}{2 n+1} .
$$

On the other hand, by using the fact that

$$
\int_{C_{\gamma}} t^{b} d t= \begin{cases}2 \pi i & (b=-1) \\ \gamma^{b+1}\left(\frac{e^{2 \pi i b}-1}{b+1}\right) & (b \neq-1)\end{cases}
$$

for $b \in \mathbb{C}$ and (11), we have

$$
I_{2}= \begin{cases}(2 \pi i) \mathcal{E}_{N}(u) \frac{(-1)^{N}}{N !} & \text { (if } c=0), \\ \gamma^{c-N}\left(e^{2 \pi i c}-1\right) \sum_{n=0}^{\infty} \frac{\mathcal{E}_{n}(u)(-1)^{n} \gamma^{n}}{(n+c-N) n !} & \text { (if } 0<c<1) .\end{cases}
$$

Note that the above infinite series in the second case is convergent because of the assumption $\gamma<\pi / 2$. Hence we have

$$
\left|I_{2}\right| \leq \begin{cases}2 \pi \frac{\left|\mathcal{E}_{N}(u)\right|}{N !} & \text { (if } c=0), \\ \gamma^{c-N}\left|e^{2 \pi i c}-1\right|\left|\sum_{n=0}^{\infty} \mathcal{E}_{n}(u) \frac{(-1)^{n} \gamma^{n}}{(n+c-N) n !}\right| & \text { (if } 0<c<1) .\end{cases}
$$


For the first case, as we mentioned above, there exists a constant $M_{1}(\gamma)>0$ independent of $u$ such that

$$
\left|I_{2}\right| \leq \frac{2 \pi M_{1}(\gamma)}{\gamma^{N}}
$$

For the second case, we let $d=\min |c-m|$ for all $m \in \mathbb{Z}$. Fix $\xi$ with $0<\gamma<\xi<$ $\pi / 2$. Then we see that the second case yields that

$$
\left|I_{2}\right| \leq \gamma^{c-N}\left|e^{2 \pi i c}-1\right| \frac{M_{1}(\xi)}{d(1-\gamma / \xi)}
$$

Hence, by combining (12)-(16), there exists a constant $M_{2}=M_{2}(\gamma)>0$ which depends on $\gamma$ but is independent of $N$ and $u$ such that

$$
\left|\frac{\rho(c-N ; u)}{\Gamma(1+N-c)}\right|=\frac{1}{4 \pi}|H(c-N ; u)| \leq \frac{M_{2}(\gamma)}{\gamma^{N}} \quad\left(N \in \mathbb{N}_{0}\right) .
$$

Since $|\Gamma(1+N-c)| \leq N !|\Gamma(1-c)|$, we obtain the proof of (9). Furthermore, we can immediately obtain (10) from (9).

For $t \in \mathbb{C}, r \in \mathbb{R}$ with $r \geq 1$ and $u \in[1,1+\delta]$, we let

$$
\mathcal{F}(t ; r ; u)=\sum_{n=0}^{\infty} \frac{(-u)^{-n} e^{(2 n+1) t}}{(2 n+1)^{r}} .
$$

Here $\mathcal{F}(t ; r ; u)$ is holomorphic for $t \in \mathbb{C}$ with $\operatorname{Re}(t)<0$. Suppose $u \in(1,1+\delta]$, $\theta \in(-\pi / 2, \pi / 2), l \in \mathbb{N}$ and $c \in \mathbb{R}$ with $0 \leq c<1$, and let $r=l+c$. By (8), we have

$$
\mathcal{F}(i \theta ; l+c ; u)=\sum_{j=0}^{\infty} \rho(l+c-j ; u) \frac{(i \theta)^{j}}{j !} .
$$

It follows from Lemma 2.1 that the right-hand side of (17) is uniformly convergent with respect to $u \in[1,1+\delta]$ when $\theta \in(-\pi / 2, \pi / 2)$. Hence $\mathcal{F}(i \theta ; l+c ; 1)$ is defined by the right-hand side of (17) with $u=1$ for $\theta \in(-\pi / 2, \pi / 2)$. Thus we can define

$$
\mathcal{F}(t ; l+c ; u)=\sum_{j=0}^{\infty} \rho(l+c-j ; u) \frac{t^{j}}{j !} \quad\left(|t|<\frac{\pi}{2}\right)
$$

for $u \in[1,1+\delta]$. Putting $r=l+c \geq 1$, we obtain the following.

Lemma 2.2. Suppose $r \in \mathbb{R}$ with $r \geq 1$ and $u \in[1,1+\delta]$. Then $\mathcal{F}(t ; r ; u)$ is holomorphic on $\{t \in \mathbb{C}|| t \mid<\pi / 2\}$. 


\section{Some lemmas}

From (5), (11), (12) and (13) with $c=0$, we see that

$$
\rho(-k ; u)=\frac{1}{2} \varepsilon_{k}(u) \quad\left(k \in \mathbb{N}_{0}\right)
$$

for $u \in[1,1+\delta]$.

We denote the $p$ th derivative of $\sin (X)$ by $\sin ^{(p)}(X)$. Furthermore, we denote $\left.\sin ^{(p)}(X)\right|_{X=\alpha}$ by $\sin ^{(p)}(\alpha)$ for $\alpha \in \mathbb{R}$. Let $\lambda_{m}=\left\{1+(-1)^{m}\right\} / 2$ for $m \in \mathbb{Z}$. Then we have

$$
\sin ^{(p)}(X)=\frac{i^{p-1}}{2}\left(e^{i X}+(-1)^{p-1} e^{-i X}\right)=i^{p-1} \sum_{j=0}^{\infty} \lambda_{p+1+j} \frac{(i X)^{j}}{j !}
$$

For $k \in \mathbb{N}, p \in \mathbb{N}_{0}, u \in[1,1+\delta]$ and $\theta \in \mathbb{R}$, we define

$$
\begin{aligned}
X_{p}(i \theta ; k ; u) & =\sum_{n=0}^{\infty} \frac{(-u)^{-n} \sin ^{(p)}((2 n+1) \theta)}{(2 n+1)^{k}} \\
& =\frac{i^{p-1}}{2}\left\{\mathcal{F}(i \theta ; k ; u)+(-1)^{p-1} \mathcal{F}(-i \theta ; k ; u)\right\}
\end{aligned}
$$

and

$$
y_{p}(i \theta ; k ; u)=X_{p}(i \theta ; k ; u)-i^{p-1} \sum_{j=0}^{k} \rho(k-j ; u) \lambda_{p+1+j} \frac{(i \theta)^{j}}{j !}
$$

for $k \in \mathbb{N}, p \in \mathbb{N}_{0}, u \in[1,1+\delta]$ and $\theta \in \mathbb{R}$. When $u \in(1,1+\delta]$, it follows from (17), (19)-(22) that

$$
y_{p}(i \theta ; k ; u)=\frac{i^{p-1}}{2} \sum_{n=1}^{\infty} \mathcal{E}_{n}(u) \lambda_{p+1+n+k} \frac{(i \theta)^{n+k}}{(n+k) !} .
$$

From Lemma 2.1, this is uniformly convergent with respect to $u \in[1,1+\delta]$ when $\theta \in(-\pi / 2, \pi / 2)$. By (6), we have $\mathcal{E}_{n}(1) \lambda_{n+1}=0$ for $n \in \mathbb{N}$. Hence we obtain the following.

LEMMA 3.1. With the above notation and under the assumption that $p \equiv k(\bmod 2)$ and $\theta \in(-\pi / 2, \pi / 2)$,

$$
\lim _{u \rightarrow 1} y_{p}(i \theta ; k ; u)=0
$$


For $u \in[1,1+\delta]$, we define

$$
\begin{aligned}
\mathfrak{S}\left(s_{1}, s_{2}, s ; u\right) & :=\sum_{m, n=0}^{\infty} \frac{(-u)^{-m-n}}{(2 m+1)^{s_{1}}(2 n+1)^{s_{2}}(2 m+2 n+2)^{s}}, \\
\mathfrak{R}\left(s_{1}, s_{2}, s ; u\right) & :=\sum_{m, n=0}^{\infty} \frac{(-u)^{-2 m-n}}{(2 m+1)^{s_{1}}(2 n+2)^{s_{2}}(2 m+2 n+3)^{s}} .
\end{aligned}
$$

By [9, Lemma 2.2], we have the following.

LEMMA 3.2. For $u \in(1,1+\delta]$,

$$
\begin{aligned}
& \mathcal{F}\left(i \theta ; s_{1} ; u\right) \mathcal{F}\left(i \theta ; s_{2} ; u\right)=\sum_{N=0}^{\infty} \mathfrak{S}\left(s_{1}, s_{2},-N ; u\right) \frac{(i \theta)^{N}}{N !}, \\
& \mathcal{F}\left(-i \theta ; s_{1} ; u\right) \mathcal{F}\left(i \theta ; s_{2} ; u\right) \\
& =-\frac{1}{u} \sum_{N=0}^{\infty}\left\{\mathfrak{R}\left(s_{1},-N, s_{2} ; u\right)+(-1)^{N} \mathfrak{R}\left(s_{2},-N, s_{1} ; u\right)\right\} \frac{(i \theta)^{N}}{N !} \\
& \quad+\sum_{m=0}^{\infty} \frac{u^{-2 m}}{(2 m+1)^{s_{1}+s_{2}}} .
\end{aligned}
$$

For $n \in \mathbb{Z}, p \in \mathbb{N}_{0}, k \in \mathbb{N}, r \in \mathbb{R}$ with $r>1$ and $u \in(1,1+\delta]$, we define

$$
\begin{aligned}
\mathcal{B}^{p}(n ; k, r ; u) & \\
= & \frac{1}{2}\left[\mathfrak{S}(k, r, n ; u)+\frac{(-1)^{p}}{u}\left\{\mathfrak{R}(k, n, r ; u)+(-1)^{n} \mathfrak{R}(r, n, k ; u)\right\}\right] \\
& -\sum_{j=0}^{k}\left(\begin{array}{c}
-n \\
j
\end{array}\right) \rho(k-j ; u) \lambda_{p+1+j} \rho(r+j+n ; u) .
\end{aligned}
$$

Note that if $n \in \mathbb{N}$ then we can define $\mathcal{B}^{p}(n ; k, r ; 1)$ by the right-hand side of (29) with $u=1$.

LEMMA 3.3. With the above notation and for $u \in(1,1+\delta]$,

$$
\begin{aligned}
& y_{p}(i \theta ; k ; u) \mathcal{F}(i \theta ; r ; u) \\
& \quad=i^{p-1} \sum_{N=0}^{\infty} \mathcal{B}^{p}(-N ; k, r ; u) \frac{(i \theta)^{N}}{N !}+\frac{(-i)^{p-1}}{2} \sum_{m=0}^{\infty} \frac{u^{-2 m}}{(2 m+1)^{k+r}} .
\end{aligned}
$$

Proof. We use the same method as in the proof of [9, Lemma 2.3]. By combining (21) and Lemma 3.2 , we can calculate $X_{p}(i \theta ; k ; u) \mathcal{F}(i \theta ; r ; u)$. On the other hand, by 
combining (17) and (22) and using the binomial theorem, we have

$$
\begin{aligned}
\sum_{j=0}^{k} \rho(k-j ; u) \lambda_{p+1+j} \frac{(i \theta)^{j}}{j !} \sum_{\nu=0}^{\infty} \rho(r-\nu ; u) \frac{(i \theta)^{\nu}}{\nu !} \\
=\sum_{N=0}^{\infty}\left\{\sum_{j=0}^{k}\left(\begin{array}{c}
N \\
j
\end{array}\right) \rho(k-j ; u) \lambda_{p+1+j} \rho(r+j-N ; u)\right\} \frac{(i \theta)^{N}}{N !} .
\end{aligned}
$$

Hence, by (29), we obtain the assertion.

Lemma 3.4. Suppose $k \in \mathbb{N}, p \in \mathbb{N}_{0}, r \in \mathbb{R}$ with $r>1$ and $u \in(1,1+\delta]$. For any $\gamma \in \mathbb{R}$ with $0<\gamma<\pi / 2$, there exists a constant $\mathcal{M}=\mathcal{M}(\gamma)>0$ independent of $u \in(1,1+\delta]$ such that

$$
\frac{\left|\mathcal{B}^{p}(-n ; k, r ; u)\right|}{n !} \leq \frac{\mathcal{M}(\gamma)}{\gamma^{n}} \quad\left(n \in \mathbb{N}_{0}\right) .
$$

In particular.

$$
\liminf _{n \rightarrow \infty}\left(\frac{\left|\mathcal{B}^{p}(-n ; k, r ; u)\right|}{n !}\right)^{-1 / n} \geq \frac{\pi}{2}
$$

Furthermore, if $p \equiv k(\bmod 2)$, then

$$
\begin{aligned}
& \lim _{u \rightarrow 1} \mathcal{B}^{p}(-N ; k, r ; u)=0 \quad(N \in \mathbb{N}), \\
& \lim _{u \rightarrow 1} \mathcal{B}^{p}(0 ; k, r ; u)=\frac{(-1)^{p}}{2} \sum_{m=0}^{\infty} \frac{1}{(2 m+1)^{k+r}} .
\end{aligned}
$$

Proof. By (21), (22) and Lemma 2.2, $y_{p}(t ; k ; u)$ can be defined and holomorphic on $\{t \in \mathbb{C}|| t \mid<\pi / 2\}$ when $u \in[1,1+\delta]$. So is $y_{p}(t ; k ; u) \mathcal{F}(t ; r ; u)$. In particular, when $u \in(1,1+\delta)$, it follows from (30) that

$$
\begin{aligned}
& y_{p}(t ; k ; u) \mathcal{F}(t ; r ; u) \\
& \quad=i^{p-1} \sum_{N=0}^{\infty} \mathcal{B}^{p}(-N ; k, r ; u) \frac{t^{N}}{N !}+\frac{(-i)^{p-1}}{2} \sum_{m=0}^{\infty} \frac{u^{-2 m}}{(2 m+1)^{k+r}}
\end{aligned}
$$

for $t \in \mathbb{C}$ with $|t|<\pi / 2$. By the same method as in the proof of Lemma 2.1, we obtain (31) and (32). Hence the right-hand side of (35) is uniformly convergent with respect to $u \in(1,1+\delta$ ], namely (35) holds for $u=1$. On the other hand, by Lemma 3.1, we see that the left-hand side of $(35)$ tends to 0 as $u \rightarrow 1$, when $p \equiv k(\bmod 2)$. Thus we obtain (33) and (34). 


\section{Double series $\mathcal{T}_{a, b}\left(s_{1}, s_{2} ; s\right)$}

By the same method as in [7], we aim to give some relation formulas for $\mathfrak{T}_{a, b}\left(s_{1}, s_{2} ; s\right)$ defined by (2).

Lemma 4.1. Suppose $k \in \mathbb{N}, p \in \mathbb{N}_{0}, r \in \mathbb{R}$ with $r>1, d \in \mathbb{N}, \theta \in \mathbb{R}$ and $u \in(1,1+\delta]$. Then

$$
\begin{aligned}
& \frac{1}{2} \sum_{m, n=0}^{\infty}\{ \frac{(-u)^{-m-n} \cos ((2 m+2 n+2) \theta)}{(2 m+1)^{k}(2 n+1)^{r}(2 m+2 n+2)^{d}} \\
&+\frac{(-1)^{p}}{u} \frac{(-u)^{-2 m-n} \cos ((2 n+2) \theta)}{(2 m+1)^{k}(2 n+2)^{d}(2 m+2 n+3)^{r}} \\
&\left.+\frac{(-1)^{p+d}}{u} \frac{(-u)^{-2 m-n} \cos ((2 n+2) \theta)}{(2 m+1)^{r}(2 n+2)^{d}(2 m+2 n+3)^{k}}\right\} \\
&-\sum_{j=0}^{k} \rho_{1}(k-j ; u)(-1)^{j} \lambda_{p+1+j} \sum_{v=0}^{j} \frac{(-\theta)^{\nu}}{v !}\left(\begin{array}{c}
d-1+j-v \\
j-v
\end{array}\right) \times \sum_{m=0}^{\infty} \frac{(-u)^{-m} \cos ^{(\nu)}((2 m+1) \theta)}{(2 m+1)^{d+j+r-v}} \\
&=\sum_{N=0}^{\infty} \mathcal{B}^{p}(d-2 N ; k, r ; u) \frac{(i \theta)^{2 N}}{(2 N) !} .
\end{aligned}
$$

ProOF. By (25) and (26), we have

$$
\sum_{m, n=0}^{\infty} \frac{(-u)^{-m-n} \cos ((2 m+2 n+2) \theta)}{(2 m+1)^{s_{1}}(2 n+1)^{s_{2}}(2 m+2 n+2)^{s_{3}}}=\sum_{N=0}^{\infty} \mathfrak{S}\left(s_{1}, s_{2}, s_{3}-2 N ; u\right) \frac{(i \theta)^{2 N}}{(2 N) !},
$$

and

$$
\sum_{m, n=0}^{\infty} \frac{(-u)^{-2 m-n} \cos ((2 n+1) \theta)}{(2 m+1)^{s_{1}}(2 n+2)^{s_{2}}(2 m+2 n+3)^{s_{3}}}=\sum_{N=0}^{\infty} \Re\left(s_{1}, s_{2}-2 N, s_{3} ; u\right) \frac{(i \theta)^{2 N}}{(2 N) !}
$$

Furthermore, we recall

$$
\begin{aligned}
& \sum_{\nu=0}^{c} \frac{(-\theta)^{\nu}}{\nu !}\left(\begin{array}{c}
a+c-v \\
c-v
\end{array}\right) \sum_{m=0}^{\infty} \frac{(-u)^{-m} \cos ^{(\nu)}((2 m+b) \theta)}{(2 m+b)^{a+1+c+r-\nu}} \\
& =\sum_{N=0}^{\infty}\left(\begin{array}{c}
a+c-2 N \\
c
\end{array}\right) \sum_{m=0}^{\infty} \frac{(-u)^{-m}}{(2 m+b)^{a+1+c+r-2 N}} \frac{(i \theta)^{2 N}}{(2 N) !}
\end{aligned}
$$


for $a, c \in \mathbb{N}_{0}, b \in\{1,2\}$ and $r \in \mathbb{R}$ with $r>1$ (see [8, (3.2)]). By applying this equation with $(a, b, c)=(d-1,1, j)$, we have

$$
\begin{gathered}
(-1)^{j} \sum_{\nu=0}^{j} \frac{(-\theta)^{\nu}}{v !}\left(\begin{array}{c}
d-1+j-v \\
j-v
\end{array}\right) \sum_{m=0}^{\infty} \frac{(-u)^{-m} \cos ^{(\nu)}((2 m+1) \theta)}{(2 m+1)^{d+j+r-\nu}} \\
\quad=\sum_{N=0}^{\infty}\left(\begin{array}{c}
2 N-d \\
j
\end{array}\right) \rho(d+j+r-2 N ; u) \frac{(i \theta)^{2 N}}{(2 N) !}
\end{gathered}
$$

because

$$
\left(\begin{array}{c}
d-1+j-2 N \\
j
\end{array}\right)=(-1)^{j}\left(\begin{array}{c}
2 N-d \\
j
\end{array}\right)
$$

By (29), we obtain the assertion.

When $d \geq 2$ and $\theta \in(-\pi / 2, \pi / 2)$, it follows from (31) that there exists a constant $M>1$ independent of $u \in(1,1+\delta]$ such that

$$
\left|\mathcal{B}^{p}(d-2 N ; k, r ; u) \frac{(i \theta)^{2 N}}{(2 N) !}\right| \leq M \frac{(2 N-d) !}{2 N !}\left(\frac{\pi}{2}\right)^{d} \leq \frac{M}{(2 N-1)^{2}}\left(\frac{\pi}{2}\right)^{d}
$$

for any $N$ with $2 N \geq d$. This means that if $d \geq 2$ then the right-hand sides of (36) is uniformly convergent with respect to $u \in(1,1+\delta]$. So we can let $u \rightarrow 1$ in both sides of (36), namely (36) holds for $u=1$ when $\theta \in(-\pi / 2, \pi / 2)$. Using Lemma 3.4, we have the following proposition. Note that each side of (37) is continuous for $\theta \in[-\pi / 2, \pi / 2]$, hence (37) holds for $\theta \in[-\pi / 2, \pi / 2]$.

PROPOSITION 4.2. Suppose $k \in \mathbb{N}, p \in \mathbb{N}_{0}, r \in \mathbb{R}$ with $r>1, d \in \mathbb{N}$ with $d \geq 2$ and $\theta \in[-\pi / 2, \pi / 2]$. If $k \equiv p(\bmod 2)$, then

$$
\begin{aligned}
& \frac{1}{2} \sum_{m . n=0}^{\infty}\{ \frac{(-1)^{m+n} \cos ((2 m+2 n+2) \theta)}{(2 m+1)^{k}(2 n+1)^{r}(2 m+2 n+2)^{d}} \\
&+(-1)^{p} \frac{(-1)^{n} \cos ((2 n+2) \theta)}{(2 m+1)^{k}(2 n+2)^{d}(2 m+2 n+3)^{r}} \\
&\left.+(-1)^{p+d} \frac{(-1)^{n} \cos ((2 n+2) \theta)}{(2 m+1)^{r}(2 n+2)^{d}(2 m+2 n+3)^{k}}\right\} \\
&-\sum_{j=0}^{k} \rho(k-j ; 1)(-1)^{j} \lambda_{p+1+j} \sum_{v=0}^{j} \frac{(-\theta)^{v}}{v !}\left(\begin{array}{c}
d-1+j-v \\
j-v
\end{array}\right) \times \sum_{m=0}^{\infty} \frac{(-1)^{m} \cos ^{(v)}((2 m+1) \theta)}{(2 m+1)^{d+j+r-v}} \\
&=\sum_{N=0}^{|d / 2|} \mathcal{B}^{p}(d-2 N ; k, r ; 1) \frac{(i \theta)^{2 N}}{(2 N) !} .
\end{aligned}
$$


When $d \geq 3$, we can differentiate (37) with respect to $\theta$ because of its uniform convergency. Using the known relation

$$
-\left(\begin{array}{l}
x-1 \\
y-1
\end{array}\right)+\left(\begin{array}{l}
x \\
y
\end{array}\right)=\left(\begin{array}{c}
x-1 \\
y
\end{array}\right)
$$

we have the following.

PROPOSITION 4.3. Suppose $k \in \mathbb{N}, p \in \mathbb{N}_{0}, r \in \mathbb{R}$ with $r>1, d \in \mathbb{N}$ with $d \geq 3$ and $\theta \in[-\pi / 2, \pi / 2]$. If $k \equiv p(\bmod 2)$, then

$$
\begin{aligned}
&-\frac{1}{2} \sum_{m, n=0}^{\infty}\{ \frac{(-1)^{m+n} \sin ((2 m+2 n+2) \theta)}{(2 m+1)^{k}(2 n+1)^{r}(2 m+2 n+2)^{d-1}} \\
&+(-1)^{p} \frac{(-1)^{n} \sin ((2 n+2) \theta)}{(2 m+1)^{k}(2 n+2)^{d-1}(2 m+2 n+3)^{r}} \\
&\left.+(-1)^{p+d} \frac{(-1)^{n} \sin ((2 n+2) \theta)}{(2 m+1)^{r}(2 n+2)^{d-1}(2 m+2 n+3)^{k}}\right\} \\
&-\sum_{j=0}^{k} \rho(k-j ; 1)(-1)^{j} \lambda_{p+1+j} \sum_{\nu=0}^{j} \frac{(-\theta)^{v}}{\nu !}\left(\begin{array}{c}
d-2+j-v \\
j-v
\end{array}\right) \quad \times \sum_{m=0}^{\infty} \frac{(-1)^{m} \cos ^{(\nu+1)}((2 m+1) \theta)}{(2 m+1)^{d-1+j+r-\nu}} \\
&=\sum_{N=1}^{[d / 2\}} \mathcal{B}^{p}(d-2 N ; k, r ; 1) \frac{(-1)^{N} \theta^{2 N-1}}{(2 N-1) !} .
\end{aligned}
$$

We apply Proposition 4.2 and Proposition 4.3 with $\theta=\pi / 2$. In particular, in (39) we replace $d-1$ with $d$. Let $\psi(s)=\sum_{n \geq 0}(2 n+1)^{-s}=\left(1-2^{-s}\right) \zeta(s)$. Then we have the following relations for $\mathcal{T}_{a, b}\left(s_{1}, s_{2} ; s\right)$.

COROLlaRY 4.4. Suppose $k \in \mathbb{N}, p \in \mathbb{N}_{0}, r \in \mathbb{R}$ with $r>1$ and $d \in \mathbb{N}$ with $d \geq 2$. If $k \equiv p(\bmod 2)$, then

(40) $\frac{1}{2}\left\{-\mathfrak{T}_{1,1}(k, r ; d)+(-1)^{p+1} \mathfrak{T}_{1,2}(k, d ; r)+(-1)^{p+1+d} \mathfrak{T}_{1,2}(r, d ; k)\right\}$

$$
\begin{aligned}
& -\sum_{j=0}^{k} \rho(k-j ; 1)(-1)^{j} \lambda_{p+1+j} \\
& \times \sum_{\mu=0}^{[(j-1) / 2]} \frac{(-1)^{\mu}(\pi / 2)^{2 \mu+1}}{(2 \mu+1) !}\left(\begin{array}{c}
d-2+j-2 \mu \\
j-2 \mu-1
\end{array}\right) \psi(d+j+r-2 \mu-1) \\
& =\sum_{N=0}^{[d / 2]} \mathcal{B}^{p}(d-2 N ; k, r ; 1) \frac{(i \pi / 2)^{2 N}}{(2 N) !} .
\end{aligned}
$$


COROllaRY 4.5. Suppose $k \in \mathbb{N}, p \in \mathbb{N}_{0}, r \in \mathbb{B}$ with $r>1$ and $d \in \mathbb{N}$ with $d \geq 2$. If $k \equiv p(\bmod 2)$, then

$$
\begin{aligned}
\sum_{j=0}^{k} \rho(k-j ; 1)(-1)^{j} \lambda_{p+1+j} \\
\quad \times \sum_{\mu=0}^{|j / 2|} \frac{(i \pi / 2)^{2 \mu}}{(2 \mu) !}\left(\begin{array}{c}
d-1+j-2 \mu \\
j-2 \mu
\end{array}\right) \psi(d+j+r-2 \mu) \\
=\sum_{N=1}^{|(d+1) / 2|} \mathcal{B}^{p}(d+1-2 N ; k, r ; 1) \frac{(-1)^{N}(\pi / 2)^{2 N-1}}{(2 N-1) !} \\
=\sum_{N=0}^{\mid(d-1) / 2\}} \mathcal{B}^{p}(d-1-2 N ; k, r ; 1) \frac{(-1)^{N+1}(\pi / 2)^{2 N+1}}{(2 N+1) !} .
\end{aligned}
$$

Now we give the explicit relation formulas for $\mathfrak{T}_{a, b}\left(s_{1}, s_{2} ; s\right)$. We make use of the following lemma which is equivalent to [7, Lemma 4.4].

LEMMA 4.6. Let $\left\{\boldsymbol{A}_{2 h}\right\}_{h \in \mathbb{N}_{0}},\left\{\boldsymbol{B}_{2 h}\right\}_{h \in \mathbb{N}_{0}}$ and $\left\{\boldsymbol{C}_{2 h}\right\}_{h \in \mathbb{N}_{0}}$ be sequences such that

$$
\boldsymbol{A}_{2 h}=\sum_{j=0}^{h} \boldsymbol{C}_{2 h-2 j} \frac{(i \pi / 2)^{2 j}}{(2 j) !}, \quad \boldsymbol{B}_{2 h}=\sum_{j=0}^{h} \boldsymbol{C}_{2 h-2 j} \frac{(i \pi / 2)^{2 j}}{(2 j+1) !} \quad\left(h \in \mathbb{N}_{0}\right)
$$

Then

$$
\boldsymbol{A}_{2 h}=-2^{1-2 h} \sum_{v=0}^{h} 2^{2 v} \boldsymbol{B}_{2 v} \zeta(2 h-2 v) \quad\left(h \in \mathbb{N}_{0}\right)
$$

Proof. Putting $\left(\alpha_{2 j}, \beta_{2 j}, \gamma_{2 j}\right)=\left(2^{-2 j} \boldsymbol{A}_{2 j}, 2^{-2 j} B_{2 j}, 2^{-2 j} C_{2 j}\right)$ in [7, Lemma 4.4], we immediately obtain the assertion.

We simply write $\rho(s)=\rho(s ; 1)$. It is well-known that

$$
\rho(2 j+1)=\frac{(-1)^{j} \pi^{2 j+1}}{(2 j) ! 2^{2 j+2}} E_{2 j} \quad\left(j \in \mathbb{N}_{0}\right)
$$

(see [1, Chapter 1]). Then, from Corollary 4.4, Corollary 4.5 and Lemma 4.6, we obtain the following result. 
THEOREM 4.7. Let $k, l \in \mathbb{N}$. Then

$$
\begin{aligned}
& -\mathfrak{T}_{1,1}(k, s ; 2 l)+(-1)^{k+1}\left\{\mathfrak{T}_{1,2}(k, 2 l ; s)+\mathfrak{T}_{1,2}(s, 2 l ; k)\right\} \\
& =2 \sum_{\substack{j=0 \\
j \neq k}}^{k} \rho(k-j)(-1)^{j} \\
& \times \sum_{\mu=0}^{[(j-1) / 2]} \frac{(-1)^{\mu}(\pi / 2)^{2 \mu+1}\left(\begin{array}{c}
2 l-2+j-2 \mu \\
j-2 \mu-1
\end{array}\right)}{(2 \mu+1) !} \psi(2 l+j+s-2 \mu-1) \\
& +\frac{2^{3-2 l}}{\pi} \sum_{\substack{j=0 \\
j \neq t(\operatorname{mat} 2)}}^{k} \rho(k-j)(-1)^{j} \sum_{\mu=0}^{[j / 2]} \frac{(-1)^{\mu}(\pi / 2)^{2 \mu}}{(2 \mu) !} \\
& \times \sum_{v=1}^{l} 2^{2 v}\left(\begin{array}{c}
2 v+j-2 \mu \\
j-2 \mu
\end{array}\right) \psi(2 v+1+j+s-2 \mu) \zeta(2 l-2 v) \\
& +(-1)^{k+1} 2^{1-2 l} \psi(k+s) \zeta(2 l)
\end{aligned}
$$

and

$$
\begin{aligned}
&-\mathfrak{T}_{1,1}(k, s ; 2 l+1)+(-1)^{k+1}\left\{\mathfrak{T}_{1,2}(k, 2 l+1 ; s)-\mathfrak{T}_{1,2}(s, 2 l+1 ; k)\right\} \\
&=2 \sum_{\substack{j=0 \\
j \neq k}}^{k} \rho(k-j)(-1)^{j} \\
& \times \sum_{\substack{(\bmod 2) \\
(j-1) / 2]}} \frac{(-1)^{\mu}(\pi / 2)^{2 \mu+1}\left(\begin{array}{c}
2 l-1+j-2 \mu \\
j-2 \mu-1
\end{array}\right)}{(2 \mu+1) !} \psi(2 l+j+s-2 \mu) \\
&+ \frac{2^{3-2 l}}{\pi} \sum_{\substack{j=0 \\
j \neq k}}^{k} \rho(k-j)(-1)^{j} \sum_{\mu=0}^{[j / 2]} \frac{(-1)^{\mu}(\pi / 2)^{2 \mu}}{(2 \mu) !} \\
& \times \sum_{\nu=0}^{l} 2^{2 v}\left(\begin{array}{c}
2 \nu+1+j-2 \mu \\
j-2 \mu
\end{array}\right) \psi(2 \nu+2+j+s-2 \mu) \zeta(2 l-2 \nu)
\end{aligned}
$$

hold for all $s \in \mathbb{C}$ with both $\operatorname{Re}(s) \geq 0$ and $\operatorname{Re}(s)>1-k$, where $\psi(s)=$ $\left(1-2^{-s}\right) \zeta(s)$.

Proof. In Lemma 4.6, let $A_{2 h}$ be the left-hand side of (40) in the case $d=2 h$, $\boldsymbol{B}_{2 h}$ the left-hand side of (41) in the case $d=2 h+1$ divided by $-\pi / 2$ for $h \in \mathbb{N}$. Furthermore, let $C_{2 h}=\mathcal{B}^{k}(2 h ; k ; r ; 1)$ for $h \in \mathbb{N}$ and

$$
A_{0}=B_{0}=C_{0}=\mathcal{B}^{k}(0 ; k, r ; 1)=\frac{(-1)^{k}}{2} \psi(k+r)
$$


(see (34)). Then, applying Lemma 4.6 and putting $h=l \in \mathbb{N}$, we see that (43) holds for any $s=r \in \mathbb{R}$ with $r>1$. We can easily check that (2) is convergent absolutely when $\operatorname{Re}\left(s_{1}\right) \geq 0, \operatorname{Re}\left(s_{2}\right) \geq 0, \operatorname{Re}(s) \geq 0$, and $\operatorname{Re}\left(s_{1}+s\right)>1, \operatorname{Re}\left(s_{2}+s\right)>1$, $\operatorname{Re}\left(s_{1}+s_{2}+s\right)>2$ (see [2,6]). Hence (43) holds for any $s \in \mathbb{C}$ with $\operatorname{Re}(s) \geq 0$ and $k+\operatorname{Re}(s)>1$. Similarly, let $\boldsymbol{A}_{2 h}$ be the left-hand side of (40) in the case $d=2 h+1$ for $h \in \mathbb{N}, B_{2 h}$ the left-hand side of (41) in the case $d=2 h+2$ divided by $-\pi / 2$ for $h \in \mathbb{N}_{0}$. Furthermore, $C_{2 h}=\mathcal{B}^{k}(2 h+1 ; k ; r ; 1)$ for $h \in \mathbb{N}_{0}$. Then, applying Lemma 4.6, putting $h=l \in \mathbb{N}$, and using the same consideration as above, we obtain (44).

EXAMPLE 1. Applying (44) with $(k, l)=(2,1)$, we obtain (3). Applying (43) with $(k, l)=(1,1)$ and replacing $s$ with $s+2$, we have

$$
\begin{aligned}
& -\mathfrak{T}_{1,1}(1, s+2 ; 2)+\mathfrak{T}_{1,2}(1,2 ; s+2)+\mathfrak{T}_{1.2}(s+2,2 ; 1) \\
& =\frac{1}{2}\left(1-2^{-s-3}\right) \zeta(s+3) \zeta(2)-\left(1-2^{-s-5}\right) \zeta(s+5) .
\end{aligned}
$$

This means that the left-hand side of (45) can be continued meromorphically to the whole complex plane. Combining (3) and (45), we have

$$
\begin{aligned}
\mathfrak{T}_{1.1}(2, s ; 3)+\mathfrak{T}_{1.2}(2,3 ; s)-\mathfrak{T}_{1.2}(s, 3 ; 2) & \\
& -4\left\{-\mathfrak{T}_{1,1}(1, s+2 ; 2)+\mathfrak{T}_{1.2}(1,2 ; s+2)+\mathfrak{T}_{1,2}(s+2,2 ; 1)\right\} \\
= & \frac{1}{2}\left(1-2^{-s-3}\right) \zeta(s+3) \zeta(2) .
\end{aligned}
$$

From the functional equation for $\zeta(s)$, we can trivially obtain the functional equation for the left-hand side of (46).

REMARK. From Theorem 4.7, we obtain the fact that $\mathfrak{T}_{1,1}(k, m ; n)$ can be expressed as a rational linear combination of products of the Riemann zeta values, when $k, m, n \in$ $\mathbb{N}$ with $n \geq 2$ and $k+m+n$ is odd. Indeed, applying (43) with $s=m \in \mathbb{N}$ such that $k \not \equiv m(\bmod 2)$, we see that

$$
-\mathfrak{T}_{1,1}(k, m ; 2 l)+(-1)^{m}\left\{\mathfrak{T}_{1,2}(k, 2 l ; m)+\mathfrak{T}_{1,2}(m, 2 l ; k)\right\}
$$

can be expressed as a rational linear combination of products of the Riemann zeta values, by (42). Replacing $k$ with $m$ and $s$ with $k$ in (43), we also see that so is

$$
-\mathfrak{T}_{1,1}(m, k ; 2 l)+(-1)^{m+1}\left\{\mathfrak{T}_{1,2}(m, 2 l ; k)+\mathfrak{T}_{1,2}(k, 2 l ; m)\right\} .
$$

Hence, so is $\mathfrak{T}_{1.1}(k, m ; 2 l)$. Furthermore, applying (44) with $s=m \in \mathbb{N}$ such that $k \equiv m(\bmod 2)$, we see that so is $\mathfrak{T}_{1.1}(k, m ; 2 l+1)$. Thus we obtain the assertion. This fact is a special case of known results for multiple series (see [10, Theorem 1.1]). So we can regard Theorem 4.7 as analytic generalizations of the discrete results for double series given in $[9,10]$. 


\section{Acknowledgements}

The author wishes to express his sincere gratitude to Professor Kohji Matsumoto for teaching him various information about the recent work related to the multiple zeta functions and giving him valuable comments. The author also thanks to the referee for his (or her) important suggestions about the result in Section 2.

\section{References}

[1] K. Dilcher, Zeros of Bernoulli, generalized Bernoulli and Euler polynomials, Mem. Amer. Math. Soc. 386 (Amer. Math. Soc., Providence, RI, 1988).

[2] J. G. Huard, K. S. Williams and Z. Nan-Yue, 'On Tornheim's double series', Acta Arith. 75 (1996), $105-117$.

[3] K. Matsumoto, 'On Mordell-Tornheim and other multiple zeta-functions', in: Proceedings of the Session in analytic number theory and Diophantine equations. Bonn, January-June 2002 (eds. D. R. Heath-Brown and B. Z. Moroz), Bonner Mathematische Schriften 360 (Bonn, 2003) pp. 17.

[4] L. J. Mordell, 'On the evaluation of some multiple series', J. London Math. Soc. 33 (1958), 368-371.

[5] M. V. Subbarao and R. Sitaramachandrarao, 'On some infinite series of L. J. Mordell and their analogues', Pacific J. Math. 119 (1985), 245-255.

[6] L. Tornheim, 'Harmonic double series', Amer. J. Math. 72 (1950), 303-314.

[7] H. Tsumura, 'On functional relations between the Mordell-Tornheim double zeta functions and the Riemann zeta function', preprint.

[8] _ 'On some combinatorial relations for Tornheim's double series', Acta Arith. 105 (2002), $239-252$.

[9] _ 'On alternating analogues of Tornheim's double series', Proc. Amer. Math. Soc. 131 (2003), 3633-3641.

[10] _ , 'Multiple harmonic series related to multiple Euler numbers', J. Number Theory 106 (2004), 155-168.

[11] L. C. Washington, Introduction to the cyclotomic fields, 2nd edition (Springer, New York, 1997).

Department of Mathematics

Tokyo Metropolitan University

1-1, Minami-Osawa, Hachioji

Tokyo 192-0397

Japan

e-mail: tsumura@comp.metro-u.ac.jp 